\title{
CANNABINOIDS INHIBIT CALCIUM UPTAKE BY BRAIN SYNAPTOSOMES ${ }^{1}$
}

\author{
R. ADRON HARRIS ${ }^{2}$ AND JAMES A. STOKES
}

The Harry S Truman Memorial Veterans Hospital and the Department of Pharmacology, University of Missouri Health Sciences Center, Columbia, Missouri 65212

Received July 30, 1981; Revised November 5, 1981; Accepted November 25, 1981

\begin{abstract}
The depolarization-dependent uptake of calcium was studied in synaptosomes isolated from whole mouse brain and regions of rat brain. In vitro addition of $\Delta^{9}$-tetrahydrocannabinol (THC) inhibited calcium uptake. The brainstem was most sensitive to this effect-a $10^{-8} \mathrm{M}$ THC concentration was sufficient to inhibit uptake by synaptosomes from this region. Cannabidiol produced effects similar to those of THC. Cannabinol was inactive. At a concentration of $10^{-5} \mathbf{M}$, THC markedly inhibited calcium uptake, enhanced calcium efflux, and decreased the synaptosomal membrane potential. These results suggest that this concentration of THC damages synaptosomal membranes. Since lower concentrations of THC did not affect membrane potential or calcium efflux, they apparently inhibit calcium uptake through a direct action on calcium channels. We suggest that inhibition of synaptosomal calcium uptake may be involved in the anticonvulsant action of cannabinoids.
\end{abstract}

$\Delta^{9}$-Tetrahydrocannabinol (THC) produces diverse neurochemical effects. In vitro addition of the drug inhibits brain synaptosomal uptake of norepinephrine, dopamine, serotonin, $\gamma$-aminobutyric acid, and choline (Banerjee et al., 1975; Johnson et al., 1976; Lindamood and Colasanti, 1980; Hershkowitz et al., 1977), facilitates the release of norepinephrine and serotonin (Johnson et al., 1976), and inhibits the activity of ATPases and other membrane-bound brain enzymes (Hershkowitz et al., 1977; Mellors, 1979). Whether these neurochemical actions are responsible for any of the behavioral or physiological effects of the drug is not clear. Such effects are produced only by high drug concentrations: 1 to $150 \mu \mathrm{M}$ was used in the studies cited above, whereas plasma concentrations of 0.001 to $0.1 \mu \mathrm{M}$ are associated with behavioral and physiological effects (Wall and Brine, 1979). Morphological studies have shown that in vitro exposure to $50 \mu \mathrm{M}$ THC disrupts synaptosomal membranes (Hershkowitz et al., 1977) and some of the observed effects on synaptosomal function may be due to

\footnotetext{
This work was supported by funds from the Medical Research Service of the Veterans Administration and Public Health Service Grant DA-02855. We thank Gary Sieckman and Dave Reedy for assisting with the experiments, the Research Technology Branch of the National Institute on Drug Abuse for supplying the cannabinoids, and Velma Henthorne for preparing the manuscript.

${ }^{2}$ To whom correspondence should be addressed at Department of Pharmacology, University of Missouri Health Sciences Center, Columbia, MO 65212.
}

membrane damage. Nonspecific effects also are suggested by the observation that the nonpsychoactive cannabinoid, cannabidiol, is at least as potent as THC in affecting neurotransmitter uptake, membrane enzymes, and synaptosomal morphology (Hershkowitz et al., 1977; Banerjee et al., 1975; Lindamood and Colasanti, 1980).

There are many pharmacological similarities between THC and sedative hypnotics such as alcohols and barbiturates-all produce ataxia, hypothermia, and anticonvulsant effects and fluidize membrane lipids (Karler and Turkanis, 1979; Bach et al., 1976; Lawrence and Gill, 1975; Harris and Schroeder, 1981a, b). In addition, there is cross-tolerance and cross-dependence among THC, ethanol, and pentobarbital (Newman et al., 1972, 1974; Gildea and Bourn, 1977). Since inhibition of synaptosomal calcium uptake appears to be an important neurochemical effect of alcohols and barbiturates (Blaustein and Ector, 1975; Elrod and Leslie, 1980; Harris and Hood, 1980; Harris, 1981), we postulated that cannabinoids also might alter synaptosomal calcium transport. We studied the effects of in vitro addition of cannabinoids on the influx and efflux of ${ }^{45} \mathrm{Ca}$ from nondepolarized and depolarized brain synaptosomes. In addition, we evaluated the effects of THC on synaptosomal integrity by estimating membrane potentials. In these studies, we used the lipophilic cation tetraphenylphosphonium (TPP). The accumulation of TPP by synaptosomes and neuroblastoma cells is proportional to the membrane potential (Lichtschtein et al., 1979).

Interpretation of our results was facilitated by com- 
paring the effects of four cannabinoids: THC and 11-OHTHC, which produce similar behavioral and anticonvulsant effects; cannabidiol, an effective anticonvulsant with no other behavioral or physiological effects; and cannabinol, a very weak anticonvulsant that is otherwise inactive (Karler and Turkanis, 1979; Hollister, 1973; PerezReyes et al., 1973).

\section{Materials and Methods}

Animals. Male BALB/C mice were obtained from Harlan Laboratories (Indianapolis, IN) and male Sprague-Dawley rats were purchased from Charles River Laboratories (Wilmington, MA).

Materials. THC, 11-OH-THC, cannabidiol, and cannabinol were obtained from the National Institute on Drug Abuse (Bethesda, MD). The drugs were dissolved in dimethyl sulfoxide (DMSO) at a concentration of 10 mM, protected from light, and stored under $\mathrm{N}_{2}$ at $-20^{\circ} \mathrm{C}$. Aliquots of this stock solution were diluted with buffer, blended on a Vortex mixer, and sonicated immediately before each experiment. $\left[{ }^{3} \mathrm{H}\right] \mathrm{TPP}$ was kindly provided by Dr. Ronald Kaback (Roche Institute, Nutley, NJ) and nonradioactive TPP chloride was obtained from Tridom (Fluka) Chemical Co. (Hauppauge, NY). Other materials were obtained from sources indicated elsewhere (Harris and Hood, 1980).

Preparation of synaptosomes and measurement of calcium accumulation. Ficoll density gradient centrifugation was used to prepare synaptosomes from whole mouse brain and rat brain regions (Harris and Hood, 1980). Calcium accumulation and efflux by depolarized and nondepolarized synaptosomes were performed as reported previously (Harris and Hood, 1980). The uptake period was $1 \mathrm{~min}$.

For each concentration of cannabinoid, a control solution containing the appropriate amount of DMSO also was tested. Only the highest concentration $(1 \mu \mathrm{l} / \mathrm{ml}$, corresponding to $10^{-5} \mathrm{M}$ cannabinuid) of DMSO affected calcium transport. This concentration inhibited calcium uptake and enhanced calcium efflux by 10 to $20 \%$.

Measurement of TPP accumulation. Synaptosomal membrane potential was estimated by the accumulation of $\left[{ }^{3} \mathrm{H}\right] \mathrm{TPP}$ as described by Lichtschtein et al. (1979). A $0.4-\mathrm{ml}$ aliquot of synaptosomes ( 1 to $2 \mathrm{mg}$ of protein $/ \mathrm{ml}$ ) was incubated for $4 \mathrm{~min}$ at $30^{\circ} \mathrm{C}$, followed by a $1-\mathrm{min}$ incubation with $1 \mu$ l of DMSO or THC dissolved in DMSO and a 5 -min incubation with $0.1 \mathrm{ml}$ of $\left[{ }^{3} \mathrm{H}\right] \mathrm{TPP}$ (4 $\mu \mathrm{M}$ final concentration; specific activity, $400 \mathrm{Ci} / \mathrm{mol}$ ) in sodium, $5 \mu \mathrm{M}$ potassium buffer (Harris and Hood, 1980). The synaptosomes then were exposed for $5 \mathrm{~min}$ to either $0.5 \mathrm{ml}$ of a depolarizing solution $\left(65 \mathrm{mM} \mathrm{K}^{+}\right.$final concentration) or a nondepolarizing solution $\left(5 \mathrm{mM} \mathrm{K} \mathrm{K}^{+}\right.$ final concentration). Aliquots $(300 \mu \mathrm{l})$ of the solution were transferred to two $400-\mu 1$ Microfuge tubes and the synaptosomes were pelleted. The supernatant then was aspirated, the tips of the tubes were cut off, and the pellets were digested $(0.5 \mathrm{ml}$ of $0.1 \mathrm{~N} \mathrm{NaOH})$. Scintillation cocktail was added and radioactivity was determined by liquid scintillation spectrometry.

Other methods. Protein was determined by the method of Lowry et al. (1951). Drug effects were evaluated statistically by a $t$ test for paired observations.

\section{Results}

Effect of cannabinoids on calcium uptake by mouse brain synaptosomes. Several cannabinoids inhibited the depolarization-dependent uptake of calcium by mouse brain synaptosomes (Fig. 1). At $10^{-6} \mathrm{M}$, THC and cannabidiol significantly inhibited uptake, whereas a $10^{-5} \mathrm{M}$ concentration of $11-\mathrm{OH}-\mathrm{THC}$ was necessary to produce the same effect. These drugs did not alter the depolarized uptake but selectively inhibited the depolarization-dependent accumulation of calcium. Cannabinol did not affect uptake at any of the concentrations tested (Fig. 1).

Effect of cannabinoids on calcium uptake by synap. tosomes from brain regions. Synaptosomes prepared from various regions of rat brain displayed distinct sensitivity to the effects of cannabinoids. The inhibitory effects of THC were much more pronounced with synaptosomes from brainstem or cerebellum than with those from striatum or cortex (Fig. 2). For example, a THC concentration of only $10^{-8} \mathrm{M}$ inhibited calcium uptake by brainstem synaptosomes. Inhibition of brainstem calcium uptake by THC displayed a shallow dose-response curve (Fig. 2). This may reflect more than one process; the possibility that the effects of high concentrations of THC are distinct from those of low concentrations is discussed below. Cannabidiol also inhibited brainstem synaptosomal calcium uptake but did not affect uptake in other regions (Table I). Cannabinol failed to affect calcium uptake in any brain region. Both THC and cannabidiol inhibited depolarization-dependent uptake of calcium without affecting the resting uptake (Table I).

Efflux of calcium from synaptosomes. The inhibition

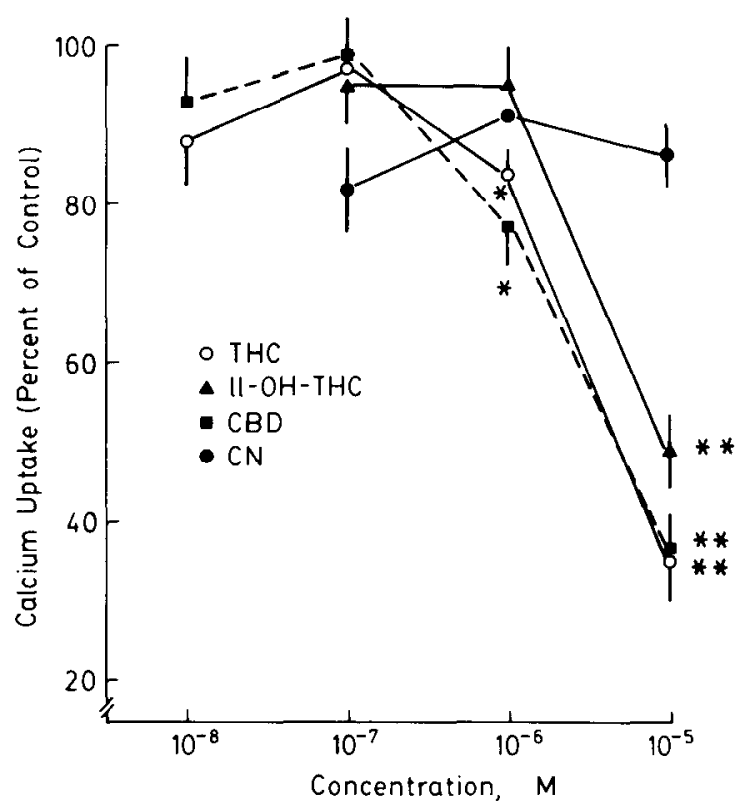

Figure 1. Effects of THC (O), 11-OH-THC (A), cannabidiol $(\square)$, and cannabinol (O) on the depolarization-dependent uptake of calcium by mouse brain synaptosomes. Drug concentrations are shown on the abscissa. The values on the ordinate represent the percentage of control uptake (calculated as the difference between depolarized and nondepolarized uptake in the absence of drug). The vertical bars signify $\pm \mathrm{SEM} ; N=4$ to 8. $\star$, Significantly different from control, $p<0.05 ; \star \star$, significantly different from control, $p<0.01$. 
of synaptosomal calcium accumulation produced by THC could result from an inhibition of calcium influx or an enhancement of calcium efflux. To distinguish between these possibilities, mouse brain synaptosomes were used to evaluate the effects of THC on calcium efflux. At $10^{-5}$ M, THC enhanced calcium efflux (Fig. 3); at $10^{-6} \mathbf{M}$, the drug did not alter efflux (data not shown). The results shown in Figure 3 were obtained by using a solution in which $\mathrm{LiCl}$ was substituted for $\mathrm{NaCl}$. This solution (lithium, $5 \mu \mathrm{M}$ potassium) minimized the sodium-calcium

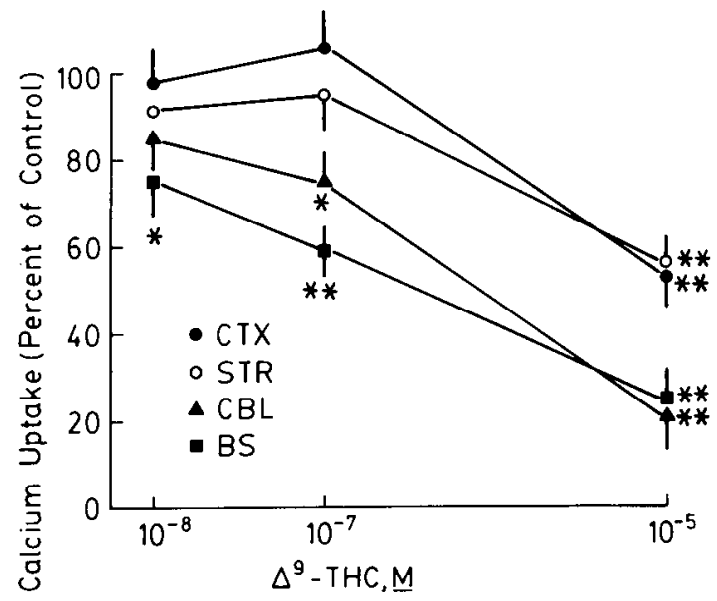

Figure 2. Effects of THC on the depolarization-dependent uptake of calcium by synaptosomes from different regions of rat brain. The symbols represent: $\boldsymbol{O}$, cortex; $\bigcirc$, striatum; $\boldsymbol{\Delta}$, cerebellum; $\mathbf{D}$, brainstem. Drug concentrations are shown on the abscissa. The values on the ordinate represent the percentage of control uptake (calculated as the difference between depolarized and nondepolarized uptake in the absence of THC). The vertical bars signify \pm SEM; $N=4$ to 6 . *, Significantly different from control, $p<0.05 ; \star \star$, significantly different from control, $p<0.01$.

TABLE I

Effects of cannabinoids on calcium uptake by synaptosomes from different regions of rat brain

Calcium Uptake

\begin{tabular}{|c|c|c|c|c|}
\hline \multirow[b]{2}{*}{ Brain Region } & \multirow[b]{2}{*}{ In Vitro Addition } & \multicolumn{3}{|c|}{ Calcium Uptake } \\
\hline & & $\begin{array}{l}\text { Nondepolar- } \\
\text { ized } \\
(-\mathrm{K})\end{array}$ & $\begin{array}{l}\text { Depolarized } \\
\qquad(+K)\end{array}$ & $\begin{array}{l}\text { Net } \\
(\Delta K)\end{array}$ \\
\hline & & \multicolumn{3}{|c|}{ nmol/mg protein } \\
\hline \multirow[t]{4}{*}{ Brainstem } & Control & $2.6 \pm 0.2^{a}$ & $5.8 \pm 0.4$ & $3.2 \pm 0.2$ \\
\hline & $\Delta^{9}-\mathrm{THC}^{b}$ & $2.6 \pm 0.3$ & $4.6 \pm 0.3^{c}$ & $1.9 \pm 0.1^{d}$ \\
\hline & Cannabidiol $^{b}$ & $2.5 \pm 0.3$ & $5.2 \pm 0.4$ & $2.7 \pm 0.2^{c}$ \\
\hline & Cannabinol $^{b}$ & $2.5 \pm 0.4$ & $5.9 \pm 0.5$ & $3.4 \pm 0.3$ \\
\hline \multirow{4}{*}{$\begin{array}{l}\text { Cerebel- } \\
\text { lum }\end{array}$} & Control & $2.9 \pm 0.2$ & $6.2 \pm 0.5$ & $3.2 \pm 0.2$ \\
\hline & $\Delta^{9}-\mathrm{THC}$ & $3.4 \pm 0.3$ & $5.8 \pm 0.6$ & $2.4 \pm 0.1^{d}$ \\
\hline & Cannabidiol & $3.5 \pm 0.4$ & $6.8 \pm 0.7$ & $3.3 \pm 0.3$ \\
\hline & Cannabinol & $3.0 \pm 0.3$ & $6.3 \pm 0.6$ & $3.3 \pm 0.2$ \\
\hline \multirow[t]{4}{*}{ Cortex } & Control & $4.1 \pm 0.3$ & $9.0 \pm 0.8$ & $4.9 \pm 0.3$ \\
\hline & $\Delta^{9}-\mathrm{THC}$ & $3.9 \pm 0.4$ & $9.1 \pm 0.9$ & $5.2 \pm 0.4$ \\
\hline & Cannabidiol & $4.3 \pm 0.5$ & $8.5 \pm 0.9$ & $4.2 \pm 0.5$ \\
\hline & Cannabinol & $4.2 \pm 0.4$ & $9.2 \pm 1.0$ & $5.0 \pm 0.4$ \\
\hline
\end{tabular}

"Values represent mean $\pm \mathrm{SEM} ; N=4$ to 6 .

${ }^{b}$ Drug concentrations were $10^{-7} \mathrm{M}$ in all cases.

' Significantly different from control, $p<0.05$.

${ }^{d}$ Significantly different from control, $p<0.01$. exchange (Blaustein and Oborn, 1975). The effects of THC were similar in a sodium-containing solution (data not shown).

THC and synaptosomal membrane potentials. The possibility that THC inhibits calcium uptake by altering membrane potentials was studied by observing $\left[{ }^{3} \mathrm{H}\right] \mathrm{TPP}$ accumulation in rat brainstem synaptosomes. TPP is a lipophilic cation, and its accumulation is proportional to membrane potential (Lichtschtein et al., 1979). The accumulation difference between depolarizing and nondepolarizing conditions $(\Delta \mathrm{K})$ reflects the plasma membrane potential, whereas the residual accumulation under depolarizing conditions $(+K)$ reflects primarily the mitochondrial membrane potential (Lichtschtein et al., 1979). At a concentration of $10^{-7} \mathrm{M}$, THC did not affect the accumulation of $\left[{ }^{3} \mathrm{H}\right] \mathrm{TPP}$, but a concentration of $10^{-5}$ $M$ markedly reduced accumulation by both depolarized and nondepolarized synaptosomes (Table II). Similar results were obtained with THC and cannabidiol in synaptosomes prepared from whole mouse brain (data not shown).

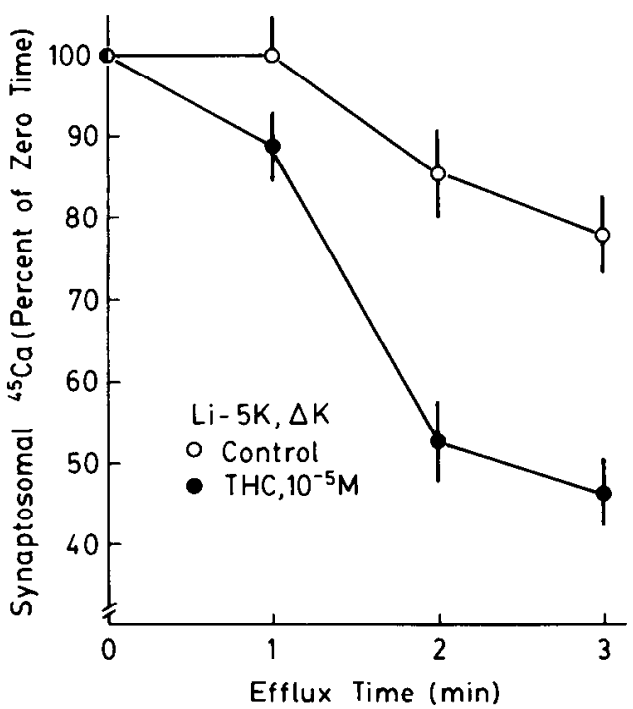

Figure 3. Effects of THC $\left(10^{-5} \mathrm{M}\right)$ on the efflux of calcium. Mouse brain synaptosomes were loaded with ${ }^{45} \mathrm{Ca}$ by depolarization and diluted with a sodium-free control $(O)$ or drug $(O)$ solution to induce calcium efflux. The abscissa represents the time after dilution (efflux time); the ordinate represents the percentage of ${ }^{45} \mathrm{Ca}$ remaining in the synaptosomes. The vertical bars signify the mean $\pm \mathrm{SEM} ; N=4$. Li-5K, Lithium, $5 \mu \mathrm{M}$ potassium.

TABLE II

Effects of THC on $\left[{ }^{3} H\right] T P P$ accumulation by synaptosomes from rat brainstem

\begin{tabular}{lccc}
\hline \multicolumn{1}{c}{ Addition } & $-\mathrm{K}$ & $+\mathrm{K}$ & $\Delta \mathrm{K}$ \\
\hline \multicolumn{3}{c}{ nmol TPP/mg protein } \\
None & $0.65 \pm 0.08^{a}$ & $0.26 \pm 0.01$ & $0.39 \pm 0.02$ \\
DMSO $^{b}$ & $0.68 \pm 0.07$ & $0.23 \pm 0.01$ & $0.45 \pm 0.02$ \\
$10^{-7} \mathrm{M} \mathrm{THC}_{10^{-5} \mathrm{M} \text { THC }}$ & $0.70 \pm 0.07$ & $0.25 \pm 0.01^{\mathrm{c}}$ & $0.45 \pm 0.03$ \\
& $0.27 \pm 0.03^{\mathrm{c}}$ & $0.15 \pm 0.01$ & $0.11 \pm 0.01^{\mathrm{c}}$ \\
\hline
\end{tabular}

${ }^{a}$ Values represent mean $\pm \mathrm{SEM} ; N=4$.

${ }^{b}$ DMSO was used as the vehicle for THC solutions. The final concentration of DMSO was $1 \mu \mathrm{l} / \mathrm{ml}$.

${ }^{c}$ Significantly different from DMSO values, $p<0.01$. 


\section{Discussion}

In our study, several cannabinoids inhibited the depolarization-dependent uptake of calcium by synaptosomes from mouse and rat brain. A regional analysis indicated that brainstem was most sensitive to the effects of THC and cannabidiol, which, at concentrations of $10^{-7}$ to $10^{-8} \mathrm{M}$, inhibited calcium transport by brainstem synaptosomes. A $10^{-7} \mathrm{M}$ concentration of THC also inhibited cerebellar synaptosomes but had no effect on striatal and cortical nerve endings. This concentration is similar to human plasma levels of THC after smoking marihuana (Wall and Brine, 1979). Synaptosomal calcium transport appears to be one of the few processes affected by nontoxic concentrations of THC.

In contrast to the inhibitory effects of THC, cannabinol did not alter calcium uptake by synaptosomes from either whole mouse brain or rat brain regions. THC and cannabidiol were equipotent in decreasing calcium uptake by mouse brain synaptosomes, but THC was more potent than cannabidiol in inhibiting uptake by brainstem and cerebellar synaptosomes. Other studies have shown that cannabidiol and cannabinol, in contrast to THC, produce few behavioral, physiological, or subjective effects (Mechoulam et al., 1970; Jarbe and Henriksson, 1974; Perez-Reyes et al., 1973; Hollister, 1973; Lemberger, 1980). Cannabidiol, however, is an effective anticonvulsant drug, whereas the anticonvulsant activity of cannabinol is weak (Karler and Turkanis, 1979, 1980). There are several similarities between the anticonvulsant action of cannabinoids and their effects on synaptosomal calcium uptake. For example, THC and cannabinol are equipotent anticonvulsants in mice, but THC is more potent in rats and is not effective against cortical (focal) seizures (Karler and Turkanis, 1979). Other anticonvulsants (e.g., phenytoin, phenobarbital, pentobarbital, and ethanol) also inhibit the depolarization-dependent uptake of calcium by brain synaptosomes (Sohn and Ferrendelli, 1976; Harris and Hood, 1980). The regional specificity of the inhibitory action of two of these drugs (ethanol and pentobarbital) is similar to that of THC, with brainstem and cerebellum being much more strongly affected than cortex or striatum (Elrod and Leslie, 1980; Harris, 1981). 'Taken together, these results suggest that inhibition of calcium uptake by nerve endings from caudal brain areas is a common feature of anticonvulsant drugs. This action of THC and cannabidiol probably is related to their anticonvulsant effects.

At a concentration of $10^{-6} \mathrm{M}$, THC did not alter the efflux of calcium from synaptosomes; therefore, at this concentration, the drug appears to reduce calcium influx through the voltage-dependent calcium channel rather than by enhancing efflux. Higher concentrations of the drug yielded markedly different results-at $10^{-5} \mathrm{M}$ THC, calcium efflux was enhanced and TPP accumulation was reduced markedly in both depolarized and nondepolarized synaptosomes. The decrease in depolarization-dependent $(\Delta \mathrm{K})$ accumulation of TPP indicates a large decrease in the potential across the plasma membrane $\left(\psi_{m}\right)$ (Lichtschtein et al., 1979). It is very likely that $10^{-5}$ M THC damages the plasma and mitochondrial membranes, whereupon they become permeable to ions. Other investigators (Hershkowitz et al., 1977) have presented morphological evidence that $3 \times 10^{-5} \mathrm{M}$ THC damages synaptosomes; therefore, it is important to note that, in our study, $10^{-7}$ to $10^{-8} \mathrm{M}$ concentrations of THC were sufficient to inhibit calcium uptake by brainstem synaptosomes, an action unlikely to have been caused by membrane damage. In contrast, $10^{-5}$ to $10^{-4} \mathrm{M}$ THC is necessary to inhibit synaptosomal uptake and stimulation of neurotransmitter release (Banerjee et al., 1975; Johnson et al., 1976).

When studying the in vitro effects of cannabinoids on neurochemical processes, it seems important to use concentrations that do not produce membrane destruction. Our results demonstrated that nontoxic concentrations of THC and cannabidiol altered synaptosomal calcium transport, which would indicate that this effect may have a bearing on the anticonvulsant action of these drugs.

\section{References}

Bach, D., A. Raz, and R. Goldman (1976) The effect of hashish compounds on phospholipid phase transition. Biochim. Biophys. Acta 436: 889-894.

Banerjee, S. P., S. H. Snyder, and R. Mechoulam (1975) Cannabinoids: Influence on neurotransmitter uptake in rat brain synaptosomes. J. Pharmacol. Exp. Ther. 194: 74-81.

Blaustein, M. P., and A. C. Ector (1975) Barbiturate inhibition of calcium uptake by depolarized nerve terminals in vitro. Mol. Pharmacol. 11: 369-378.

Blaustein, M. P., and C. J. Oborn (1975) The influence of sodium on calcium fluxes in pinched-off nerve terminals in vitro. J. Physiol. (Lond.) 247: 657-686.

Elrod, S. V., and S. W. Leslie (1980) Acute and chronic effects of barbiturates on depolarization-induced calcium influx into synaptosomes from rat brain regions. J. Pharmacol. Exp. Ther. 212: 131-136.

Gildea, M. L., and W. M. Bourn (1977) The effect of delta-9tetrahydrocannabinol on barbiturate withdrawal convulsions in the rat. Life Sci. 21: 829-832

Harris, R. A. (1981) Psychoactive drugs as antagonists of actions of calcium. In New Perspectives on Calcium Antagonists, G. Weiss, ed., pp. 223-231, American Physiological Society, Bethesda, MD.

Harris, R. A., and W. F. Hood (1980) Inhibition of synaptosomal calcium uptake by ethanol. J. Pharmacol. Exp. Ther. 213: $562-568$.

Harris, R. A., and F. Schroeder (1981a) Effects of ethanol and related drugs on the physical and functional properties of brain membranes. In Currents in Alcoholism, M. Galanter, ed., pp. 461-468, Grune and Stratton, New York.

Harris, R. A., and F. Schroeder (1981b) Ethanol and the physical properties of brain membranes: Fluorescence studies. Mol. Pharmacol. 20: 128-137.

Hershkowitz, M., R. Goldman, and A. Raz (1977) Effect of cannabinoids on neurotransmitter uptake, ATPase activity and morphology of mouse brain synaptosomes. Biochem. Pharmacol. 26: 1327-1331.

Hollister, L. E. (1973) Cannabidiol and cannabinol in man. Experientia 29: 825-826.

Jarbe, T., and B. G. Henriksson (1974) Discriminative response control produced with hashish, tetrahydrocannabinols $\left(\Delta^{8}\right.$. THC and $\Delta^{9}$-THC), and other drugs. Psychopharmacologia 40: $1-16$.

Johnson, K. M., B. T. Ho, and W. L. Dewey (1976) Effects of $\Delta^{y}$-tetrahydrocannabinol on neurotransmitter accumulation and release mechanisms in rat forebrain synaptosomes. Life Sci. 19: 347-356.

Karler, R., and S. A. Turkanis (1979) Cannabis and epilepsy. In 
Marihuana-Biological Effects, G. G. Nahas and W. D. M. Paton, eds., pp. 619-641, Pergamon Press, Elmsford, NY.

Karler, R., and S. A. Turkanis (1980) Subacute cannabinoid treatment: Anticonvulsant activity and withdrawal excitability in mice. Br. J. Pharmacol. 68: 479-484.

Lawrence, D. K., and E. W. Gill (1975) The effects of $\Delta^{1}$ tetrahydrocannabinol and other cannabinoids on spin-labeled liposomes and their relationship to mechanisms of general anesthesia. Mol. Pharmacol. 11: 595-602.

Lemberger, L. (1980) Potential therapeutic usefulness of marijuana. Annu. Rev. Pharmacol. Toxicol. 20: 151-172.

Lichtschtein, D., H. R. Kaback, and A. J. Blume (1979) Use of a lipophilic cation for determination of membrane potential in neuroblastoma-glioma hybrid cell suspension. Proc. Natl. Acad. Sci. U. S. A. 76: 650-654.

Lindamood, C., III, and B. K. Colasanti (1980) Effects of $\Delta^{9}$ tetrahydrocannabinol and cannabidiol on sodium-dependent high affinity choline uptake in the rat hippocampus. J. Pharmacol. Exp. Ther. 213: 216-221.

Lowry, O. H., N. J. Rosebrough, A. L. Farr, and R. J. Randall (1951) Protein measurement with the Folin phenol reagent. J. Biol. Chem. 193: 265-275.

Mechoulam, R., A. Shani, H. Edery, and Y. Grunfeld (1970) Chemical basis of hashish activity. Science 169: 611 .
Mellors, A. (1979) Cannabinoids and membrane-bound enzymes. In Marihuana-Biological Effects, G. G. Nahas and W. D. M. Paton, eds., pp. 329-342, Pergamon Press, Elmsford, NY.

Newman, L. M., M. P. Lutz, M. H. Gould, and E. F. Domino (1972) $\Delta^{9}$-Tetrahydrocannabinol and ethyl alcohol: Evidence for cross-tolerance in the rat. Science 175: 1022-1023.

Newman, L. M., M. P. Lutz, and E. F. Domino (1974) $\Delta^{9}$ Tetrahydrocannabinol and some CNS depressants: Evidence for cross-tolerance in the rat. Arch. Int. Pharmacodyn. 207: 254-259.

Perez-Reyes, M., M. C. Timmons, K. H. David, and E. M. Wall (1973) A comparison of the pharmacological activity in man of intravenously administered $\Delta^{9}$-tetrahydrocannabinol, cannabinol, and cannabidiol. Experientia 29: 1368-1369.

Sohn, R. S., and J. A. Ferrendelli (1976) Anticonvulsant drug mechanisms: Phenytoin, phenobarbital, and ethosuximide and calcium flux in isolated presynaptic endings. Arch. Neurol. 33: 626-629.

Wall, M. E., and D. R. Brine (1979) Applications of mass spectrometry in cannabinoid research. In Marihuana-Biological Effects, G. G. Nahas and W. D. M. Paton, eds., pp. 15-43, Pergamon Press, Elmsford, NY. 